\title{
ON AN EXISTENCE THEOREM FOR THE NAVIER-STOKES EQUATIONS WITH FREE SLIP BOUNDARY CONDITION IN EXTERIOR DOMAIN
}

\author{
RIEKO SHIMADA and NORIKAZU YAMAGUCHI \\ Department of Mathematical Sciences \\ School of Science and Engineering, Waseda University \\ 3-4-1 Ōkubo, Shinjuku-ku, Tokyo 169-8555, Japan \\ E-mail:rieko@akane.waseda.jp,norikazu@gm.math.waseda.ac.jp
}

\begin{abstract}
This paper deals with a nonstationary problem for the Navier-Stokes equations with a free slip boundary condition in an exterior domain. We obtain a global in time unique solvability theorem and temporal asymptotic behavior of the global strong solution when the initial velocity is sufficiently small in the sense of $L^{n}$ ( $n$ is dimension). The proof is based on the contraction mapping principle with the aid of $L^{p}-L^{q}$ estimates for the Stokes semigroup associated with a linearized problem, which is also discussed. In particular, we mainly discuss the local energy decay property of the semigroup which is a key estimate to prove the $L^{p}-L^{q}$ estimates in an exterior domain.
\end{abstract}

\section{Introduction and main results}

1.1. Problem. Let $\mathcal{O} \subset \mathbb{R}^{n}(n \geq 3)$ be a bounded open set with $C^{2,1}$ boundary $\partial \mathcal{O}$ and let $L_{0}$ be a positive real number such that $B_{L_{0}}(0)=\left\{x \in \mathbb{R}^{n}|| x \mid<L_{0}\right\} \supset \overline{\mathcal{O}}$. Let $\Omega$ be the exterior domain to $\mathcal{O}$, i.e., $\Omega \equiv \mathbb{R}^{n} \backslash \overline{\mathcal{O}}$. Here $\mathcal{O}$ stands for a rigid obstacle and $\Omega$ is assumed to be occupied with the viscous incompressible Newtonian fluid.

Here and in what follows, $\boldsymbol{u}=\left(u_{1}, \ldots, u_{n}\right)$ and $\pi$ stand for velocity and pressure, respectively. $\boldsymbol{n}$ stands for the unit normal vector on $\partial \Omega$. Let $\mathbf{T}(\boldsymbol{u}, \pi)$ be the stress tensor associated with flow defined by $\mathbf{T}(\boldsymbol{u}, \pi)=-2 \mathbf{D}(\boldsymbol{u})+\pi I_{n}$, where $I_{n}$ is the $n \times n$ unit matrix and $\mathbf{D}(\boldsymbol{u})=\left(\nabla \boldsymbol{u}+{ }^{t}(\nabla \boldsymbol{u})\right) / 2$ is the deformation tensor.

2000 Mathematics Subject Classification: Primary 35Q30; Secondary 76D05.

Key words and phrases: Navier-Stokes equations, free slip boundary condition, exterior domain, local energy decay.

Research of N. Yamaguchi supported by Waseda University Grant for Special Research Projects (2006-B-185). Current address: Faculty of Human Development, University of Toyama, 3190 Gofuku, Toyama-shi, Toyama 930-8555, Japan; norikazu@edu.u-toyama.ac.jp.

The paper is in final form and no version of it will be published elsewhere. 
In this paper, we are concerned with the following initial boundary value problem for the Navier-Stokes equations concerning the velocity $\boldsymbol{u}$ and pressure $\pi$ :

$$
\begin{cases}\partial_{t} \boldsymbol{u}+\boldsymbol{u} \cdot \nabla \boldsymbol{u}=\Delta \boldsymbol{u}-\nabla \pi, \quad \operatorname{div} \boldsymbol{u}=0, & x \in \Omega, t>0, \\ \boldsymbol{n} \cdot \boldsymbol{u}=0, \quad \mathbf{T}(\boldsymbol{u}, \pi)-(\boldsymbol{n} \cdot \mathbf{T}(\boldsymbol{u}, \pi) \boldsymbol{n}) \boldsymbol{n}=0, & x \in \partial \Omega, t>0, \\ \boldsymbol{u}(\cdot, 0)=\boldsymbol{a}, & x \in \Omega .\end{cases}
$$

Here $\boldsymbol{a}$ is a prescribed initial velocity and the dot '.' stands for the usual inner product in $\mathbb{R}^{n}$. We call the pair of boundary conditions in (N-S) the free slip boundary condition. This boundary condition is one of the physically reasonable boundary conditions for the motion of a viscous fluid around a rigid body. And also, in the study of the free boundary value problem of a viscous incompressible fluid, the slip boundary condition appears (see e.g., [11]).

Since $\boldsymbol{n} \cdot \boldsymbol{n}=1$, we can easily see that

$$
\mathbf{T}(\boldsymbol{u}, \pi) \boldsymbol{n}-(\boldsymbol{n} \cdot \mathbf{T}(\boldsymbol{u}, \pi) \boldsymbol{n}) \boldsymbol{n}=\mathbf{D}(\boldsymbol{u}) \boldsymbol{n}-(\boldsymbol{n} \cdot \mathrm{D}(\boldsymbol{u}) \boldsymbol{n}) \boldsymbol{n} .
$$

This relation implies that the free slip boundary condition is independent of the pressure $\pi$. This fact assures that we can use the same Helmholtz decomposition as in the case of non-slip boundary condition: $\left.\boldsymbol{u}\right|_{\partial \Omega}=0$. Hereafter, for notational simplicity, we set

$$
\begin{aligned}
\mathcal{T}(\boldsymbol{u}, \pi) & \left.\equiv(\mathbf{T}(\boldsymbol{u}, \pi) \boldsymbol{n}-(\boldsymbol{n} \cdot \mathbf{T}(\boldsymbol{u}, \pi) \boldsymbol{n}) \boldsymbol{n})\right|_{\partial \Omega} \\
& =\left.(\mathbf{D}(\boldsymbol{u}) \boldsymbol{n}-(\boldsymbol{n} \cdot \mathbf{D}(\boldsymbol{u}) \boldsymbol{n}) \boldsymbol{n})\right|_{\partial \Omega} \equiv \mathcal{D}(\boldsymbol{u}) .
\end{aligned}
$$

1.2. Known results. For the Navier-Stokes equations with slip boundary condition, Solonnikov and Ščadilov [11] studied stationary problem of the linearized equations. They showed some $L^{p}$-estimates for the solution of such problem (see Theorem 2.3 later). When $\Omega$ is bounded, Giga [2] studied the nonstationary Navier-Stokes equations with the boundary conditions: $\boldsymbol{n} \cdot \boldsymbol{u}=0$ and a general first order boundary condition including slip boundary condition. Giga showed that a sufficient condition for generation of analytic semigroup and by using fractional powers of the Stokes operator, he showed local in time existence theorem. Recently, using Giga's result Steiger [12] proved a local existence theorem and global existence theorem with very irregular initial velocity. When $\Omega$ is the half space, Saal [8] studied the resolvent problem of the Stokes equations with Navier's slip boundary condition (intermediate boundary condition of non-slip and free-slip ones). He showed the resolvent estimate and that the Stokes operator with Navier's slip boundary condition admits bounded $\mathcal{H}^{\infty}$-calculus. He also showed local in time existence of $L^{p}$-strong solutions. However, when $\Omega$ is exterior domain, as far as the authors know, there are no results about the local and global solvability for $(\mathrm{N}-\mathrm{S})$ in $L^{p}$-framework.

On the other hand, in the case of non-slip boundary condition: $\left.\boldsymbol{u}\right|_{\partial \Omega}=0$, there are many results when $\Omega$ is an exterior domain. In 1989, Iwashita [4] proved $L^{p}-L^{q}$ estimates for the Stokes semigroup generated by the Stokes operator with non-slip boundary condition and showed existence of a global solution to the initial boundary value problem of the Navier-Stokes system by using Kato's iteration scheme [5] with the aid of such $L^{p}-L^{q}$ estimates. Iwashita proved $L^{p}-L^{q}$ estimates for the Stokes semigroup by a cut-off procedure based on the $L^{p}-L^{q}$ estimates in $\mathbb{R}^{n}$ and local energy decay of the semigroup. Since the solenoidal projection and the derivative operator commute with each other in $\mathbb{R}^{n}$, the 
Stokes semigroup in $\mathbb{R}^{n}$ is essentially the same as the heat semigroup $e^{t \Delta}$. Therefore, key analysis in an exterior domain is how to get local energy decay. The local energy decay is derived from analysis of the resolvent operator near the origin. Such resolvent expansion around the origin also follows from the cut-off procedure based on the resolvent expansion in $\mathbb{R}^{n}$ and the stationary problem in a bounded domain.

Our aim in the present paper is to establish $L^{p}$-approach to (N-S) by Iwashita's argument. Since the boundary condition in (N-S) is not the homogeneous Dirichlet boundary condition, to get an asymptotic expansion of the resolvent operator near the origin, we need some modification.

1.3. Notation. To state our results precisely, we introduce notation used throughout the present paper. We set $B_{R}=\left\{x \in \mathbb{R}^{n}|| x \mid<R\right\}, \Omega_{R}=\Omega \cap B_{R}, D_{L, R}=\left\{x \in \mathbb{R}^{n} \mid\right.$ $L<|x|<R\}$.

For function spaces, we use the standard notation: $C_{0}^{\infty}(\Omega), L^{p}(\Omega)$ and $W^{m, p}(\Omega)$. For function spaces of vector fields, we use the bold letters,

$$
\begin{gathered}
\boldsymbol{L}^{p}(\Omega)=\left\{\boldsymbol{f}=\left(f_{1}, \ldots, f_{n}\right) \mid f_{j} \in L^{p}(\Omega), j=1, \ldots, n\right\}, \\
\|\boldsymbol{f}\|_{L^{p}(\Omega)}=\sum_{j=1}^{n}\left\|f_{j}\right\|_{L^{p}(\Omega)},
\end{gathered}
$$

likewise $\boldsymbol{W}^{m, p}(\Omega)$ and $\boldsymbol{C}_{0}^{\infty}(\Omega)$.

Let $X$ and $Y$ be two Banach spaces. $\mathcal{L}(X, Y)$ denotes the set of all bounded linear operators from $X$ into $Y$. If $X=Y$, we use the abbreviated form: $\mathcal{L}(X)=\mathcal{L}(X, X) \cdot \mathcal{A}(U, X)$ and $B \mathcal{A}(U, X)$ and $C^{k}(U, X)$ denote the sets of all analytic, bounded and analytic, and $C^{k}$-class functions defined on $U$ with their values in $X$, respectively. Set

$$
\begin{gathered}
U_{r}=\{z \in \mathbb{C}|| z \mid<r\}, \quad \dot{U}_{r}=U_{r} \backslash(-\infty, 0], \\
\Sigma_{\epsilon}=\{z \in \mathbb{C} \backslash\{0\}|| \arg z \mid<\pi-\epsilon\} \quad(0<\epsilon<\pi) .
\end{gathered}
$$

1.4. Stokes operator with slip boundary condition. In order to treat (N-S) as an evolution equation, here we shall introduce the Helmholtz decomposition of $\boldsymbol{L}^{p}$ vector fields. As was mentioned before, since the slip boundary condition is independent of the pressure, we can use the same Helmholtz decomposition as in the case of non-slip boundary condition.

Let $1<p<\infty$. Then $\boldsymbol{L}^{p}(\Omega)$ admits the following direct sum decomposition (see Miyakawa [7]):

$$
\boldsymbol{L}^{p}(\Omega)=L_{\sigma}^{p}(\Omega) \oplus G^{p}(\Omega)
$$

where

$$
\begin{gathered}
L_{\sigma}^{p}(\Omega)=\overline{\left\{\boldsymbol{u} \in \boldsymbol{C}_{0}^{\infty}(\Omega) \mid \operatorname{div} \boldsymbol{u}=0\right\}} \|^{\|\cdot\|_{L^{p}(\Omega)}}, \quad G^{p}(\Omega)=\left\{\nabla \pi \mid \pi \in \hat{W}^{1, p}(\Omega)\right\}, \\
\hat{W}^{1, p}(\Omega)=\left\{\pi \in L_{\mathrm{loc}}^{p}(\bar{\Omega}) \mid \nabla \pi \in \boldsymbol{L}^{p}(\Omega)\right\} .
\end{gathered}
$$

When $\Omega$ is bounded, $L_{\text {loc }}^{p}(\bar{\Omega})$ can be replaced by $L^{p}(\Omega)$ in the definition of $\hat{W}^{1, p}(\Omega)$. Moreover, when $\Omega$ is bounded $\hat{W}^{1, p}(\Omega)$ admits the following characterization: $\hat{W}^{1, p}(\Omega)=$ $W^{1, p}(\Omega) \cap\left\{\pi \in L^{p}(\Omega) \mid \int_{\Omega} \pi d x=0\right\}$.

It is well known that if $\partial \Omega \in C^{2,1}$ the solenoidal space $L_{\sigma}^{p}(\Omega)$ can be characterized as

$$
L_{\sigma}^{p}(\Omega)=\left\{\boldsymbol{u} \in \boldsymbol{L}^{p}(\Omega)|\operatorname{div} \boldsymbol{u}=0, \boldsymbol{n} \cdot \boldsymbol{u}|_{\partial \Omega}=0\right\} .
$$


Let $P=P_{p}$ be the continuous projection from $\boldsymbol{L}^{p}(\Omega)$ into $L_{\sigma}^{p}(\Omega)$ associated with (1.1). Then we define Stokes operator $A=A_{p}$ with free slip boundary condition:

$$
\begin{aligned}
A_{p} \boldsymbol{u} & =-P_{p} \Delta \boldsymbol{u} \quad \text { for } \boldsymbol{u} \in D\left(A_{p}\right), \\
D\left(A_{p}\right) & =L_{\sigma}^{p}(\Omega) \cap\left\{\boldsymbol{u} \in \boldsymbol{W}^{2, p}(\Omega) \mid \mathcal{D}(\boldsymbol{u})=0 \text { on } \partial \Omega\right\} .
\end{aligned}
$$

Note that the boundary condition $\boldsymbol{n} \cdot \boldsymbol{u}=0$ is included in $D\left(A_{p}\right)$, because of (1.2). By using $A$, we can rewrite $(\mathrm{N}-\mathrm{S})$ as an evolution equation in the Banach space $L_{\sigma}^{p}(\Omega)$ :

$(\mathrm{ACP})$

$$
\left\{\begin{array}{l}
\boldsymbol{u}^{\prime}(t)+A \boldsymbol{u}(t)=N \boldsymbol{u}(t), \quad t>0, \\
\boldsymbol{u}(0)=\boldsymbol{a},
\end{array}\right.
$$

where $N \boldsymbol{u}(t)=-P_{p}[\boldsymbol{u}(t) \cdot \nabla \boldsymbol{u}(t)]$.

Shibata and the first author [9] obtained a generalized resolvent estimate for the Stokes system with slip boundary condition. As a consequence of such generalized resolvent estimate and the Helmholtz decomposition (1.1), we have a result concerning the generation of an analytic semigroup.

Proposition 1.1. Let $1<p<\infty$. Then $-A_{p}$ generates a bounded and analytic semigroup $\left(e^{-t A_{p}}\right)_{t \geq 0}$ on $L_{\sigma}^{p}(\Omega)$.

From Duhamel's principle, (ACP) is converted into the integral equation

$$
\boldsymbol{u}(t)=e^{-t A_{p}} \boldsymbol{a}+\int_{0}^{t} e^{-(t-s) A_{p}} N \boldsymbol{u}(s) d s .
$$

Our task is to solve above integral equation by the contraction mapping principle for sufficiently small initial velocity $\boldsymbol{a} \in L_{\sigma}^{n}(\Omega)$ (see Kato [5]). To do so, we need some estimates for handling nonlinear terms in (INT).

1.5. Main results. We are now in a position to state our main results. The first result concerns $L^{p}-L^{q}$ estimates for the Stokes semigroup $e^{-t A_{p}}$, which play an essential role in our contraction mapping argument.

Theorem 1.2 ( $L^{p}-L^{q}$ estimates). For the Stokes semigroup $e^{-t A_{p}}$, the following $L^{p}-L^{q}$ estimates hold for any $\boldsymbol{a} \in L_{\sigma}^{p}(\Omega)$ and $t>0$ :

$$
\left\|\nabla^{j} e^{-t A_{p}} \boldsymbol{a}\right\|_{L^{q}(\Omega)} \leq C_{p, q} t^{-\frac{j}{2}-\frac{n}{2}\left(\frac{1}{p}-\frac{1}{q}\right)}\|\boldsymbol{a}\|_{L^{p}(\Omega)}, \quad j=0,1,
$$

where (i) $1 \leq p \leq q \leq \infty, p \neq \infty$ and $q \neq 1$ when $j=0$; (ii) $1 \leq p \leq q \leq n, q \neq 1$ when $j=1$.

From the contraction mapping principle with the aid of Theorem 1.2, we have the main result of the present paper.

THEOREM 1.3. Let $\boldsymbol{a} \in L_{\sigma}^{n}(\Omega)$. Then there exists an $\epsilon>0$ such that if $\|\boldsymbol{a}\|_{L^{n}(\Omega)} \leq \epsilon$, then $(\mathrm{N}-\mathrm{S})$ admits a unique strong solution $\boldsymbol{u} \in B C\left([0, \infty) ; L_{\sigma}^{n}(\Omega)\right)$ with

$$
\begin{gathered}
\lim _{t \rightarrow 0+0}\|\boldsymbol{u}(t)-\boldsymbol{a}\|_{L^{n}(\Omega)}=0, \\
\boldsymbol{u}(t)=o\left(t^{-\frac{1}{2}+\frac{n}{2 q}}\right), \quad n<q \leq \infty ; \quad \nabla \boldsymbol{u}(t)=o\left(t^{-\frac{1}{2}}\right), \quad t \rightarrow 0+0, \\
\boldsymbol{u}(t)=o\left(t^{-\frac{1}{2}+\frac{n}{2 q}}\right), \quad n \leq q \leq \infty ; \quad \nabla \boldsymbol{u}(t)=o\left(t^{-\frac{1}{2}}\right), \quad t \rightarrow \infty .
\end{gathered}
$$

Here $B C(I ; X)$ denotes the class of $X$-valued bounded continuous function on $I$. 
REMARK 1.4. All of our results can be extended to the case of Navier's slip boundary condition (Robin type boundary condition of non-slip and free-slip) by the same lines below (see [10]). However, in the case of Navier's slip boundary condition, we do not need any geometric assumption in Theorem 2.4 and we do not need any modification in the proof of Theorem 1.5.

In order to prove Theorem 1.2, we apply the cut-off procedure developed by Iwashita [4]. The following local energy decay of $e^{-t A}$ near the boundary plays a crucial role.

TheOrem 1.5 (local energy decay). Let $1<p<\infty$ and $R>L_{0}$. Then the following estimate holds for any $\boldsymbol{a} \in \boldsymbol{L}_{R}^{p}(\Omega)$ and $t \geq 1$ :

$$
\left\|e^{-t A} P \boldsymbol{a}\right\|_{W^{2, p}\left(\Omega_{R}\right)} \leq C_{p, R} t^{-\frac{n}{2}}\|\boldsymbol{a}\|_{L^{p}(\Omega)} .
$$

Here $\boldsymbol{L}_{R}^{p}(\Omega)=\left\{\boldsymbol{a} \in \boldsymbol{L}^{p}(\Omega) \mid \boldsymbol{a}=0\right.$ for $\left.|x|>R\right\}$.

Once we get Theorem 1.5, combining the $L^{p}-L^{q}$ estimate for the heat semigroup $e^{t \Delta}$ in $\mathbb{R}^{n}$, the resolvent estimate obtained in Shibata and Shimada [9] and Theorem 1.5 by cut-off procedure, we can obtain Theorem 1.2 (see e.g., Iwashita [4], Kobayashi and Shibata [6], Hishida [3] and Yamaguchi [13] and cited therein).

The plan of the present paper is as follows. In $\oint 2$ we will introduce classical results for the Stokes resolvent problem in $\mathbb{R}^{n}$, stationary Stokes equations when the domain is bounded and Bogovskiı's lemma which will be required later. In $\S 3$, we will give a sketch of the proof of our results, Theorems 1.2 and 1.5. First we will study the Stokes resolvent problem in an exterior domain. In particular, our main concern at this step is asymptotic behavior of the resolvent when the spectral parameter is close to the origin. After observing the asymptotic behavior of the resolvent, we shall show Theorem 1.5. Finally, we will give a sketch of the proof of Theorem 1.2.

\section{Preliminaries}

2.1. Stokes resolvent problem in $\mathbb{R}^{n}$. In this subsection, we shall give some fundamental results for the Stokes resolvent problem in $\mathbb{R}^{n}$ :

$$
\lambda \boldsymbol{u}-\Delta \boldsymbol{u}+\nabla \pi=\boldsymbol{f}, \quad \operatorname{div} \boldsymbol{u}=0, \quad x \in \mathbb{R}^{n},
$$

where $\lambda \in \mathbb{C} \backslash(-\infty, 0]$ and $\boldsymbol{f}$ is given. Set

$$
R_{0}(\lambda) \boldsymbol{f}(x) \equiv \mathcal{F}_{\xi}^{-1}\left[\frac{P_{0}(\xi) \hat{\boldsymbol{f}}(\xi)}{\lambda+|\xi|^{2}}\right](x), \quad \Pi \boldsymbol{f}(x) \equiv \mathcal{F}_{\xi}^{-1}\left[\frac{-i \xi \cdot \hat{\boldsymbol{f}}(\xi)}{|\xi|^{2}}\right](x),
$$

where $\hat{\boldsymbol{f}}$ denotes the Fourier transform of $\boldsymbol{f}, \mathcal{F}_{\xi}^{-1}[\cdot]$ stands for the inverse Fourier transform and $P_{0}(\xi)$ is an $n \times n$ matrix of the form:

$$
P_{0}(\xi)=\left(\delta_{j k}-\frac{\xi_{j} \xi_{k}}{|\xi|^{2}}\right)_{j, k=1, \ldots, n} .
$$

$\left(R_{0}(\lambda) \boldsymbol{f}, \Pi \boldsymbol{f}\right)$ solve $(2.1)$ provided $\boldsymbol{f} \in \boldsymbol{L}^{p}\left(\mathbb{R}^{n}\right)$. For operators $R_{0}(\lambda)$ and $\Pi$, the following two lemmas are well-known. 
Lemma 2.1. Let $1<p<\infty$ and $R>L_{0}$. Then for $\lambda \in \dot{U}_{3 / 4}$ and $\boldsymbol{f} \in \boldsymbol{L}_{R}^{p}\left(\mathbb{R}^{n}\right) \equiv\{\boldsymbol{f} \in$ $\boldsymbol{L}^{p}\left(\mathbb{R}^{n}\right) \mid \boldsymbol{f}=0$ for $\left.|x|>R\right\}, R_{0}(\lambda)$ has the expansion

$$
R_{0}(\lambda) \boldsymbol{f}= \begin{cases}\lambda^{\frac{n}{2}-1} G_{1}(\lambda) \boldsymbol{f}+G_{2}(\lambda) \boldsymbol{f}, & n \text { odd }, \\ \lambda^{\frac{n}{2}-1}(\log \lambda) G_{0}(\lambda) \boldsymbol{f}+\lambda^{\frac{n}{2}-1} G_{1}(\lambda) \boldsymbol{f}+G_{2}(\lambda) \boldsymbol{f}, & n \text { even, }\end{cases}
$$

where $G_{0}, G_{1} \in B \mathcal{A}\left(\dot{U}_{3 / 4}, \mathcal{L}_{p, R}\left(\mathbb{R}^{n}\right)\right)$, and $G_{2} \in \mathcal{A}\left(U_{3 / 4}, \mathcal{L}_{p, R}\left(\mathbb{R}^{n}\right)\right)$. Here and hereafter $\mathcal{L}_{p, R}\left(\mathbb{R}^{n}\right) \equiv \mathcal{L}\left(\boldsymbol{L}_{R}^{p}\left(\mathbb{R}^{n}\right), \boldsymbol{W}^{2, p}\left(\Omega_{R}\right)\right)$.

Lemma 2.2. Let $1<p<\infty$ and $R>L_{0}$. Set

$$
R_{0}(0) \equiv \mathcal{F}_{\xi}^{-1}\left[\frac{P_{0}(\xi) \hat{\boldsymbol{f}}(\xi)}{|\xi|^{2}}\right](x), \quad \boldsymbol{f} \in \boldsymbol{L}_{R}^{p}\left(\mathbb{R}^{n}\right) .
$$

Then for every $\boldsymbol{f} \in \boldsymbol{L}_{R}^{p}\left(\mathbb{R}^{n}\right),\left(R_{0}(0) \boldsymbol{f}, \Pi \boldsymbol{f}\right)$ satisfy

$$
\begin{aligned}
& \sup _{|x| \geq R+1}|x|^{n-2}\left|R_{0}(0) \boldsymbol{f}\right|+\sup _{|x| \geq R+1}|x|^{n-1}\left|\nabla R_{0}(0) \boldsymbol{f}\right|+\sup _{|x| \geq R+1}|x|^{n-1}|\Pi \boldsymbol{f}| \\
& \quad+\left\|\nabla^{2} R_{0}(0) \boldsymbol{f}\right\|_{L^{p}\left(\mathbb{R}^{n}\right)}+\left\|\left(R_{0}(0) \boldsymbol{f}, \Pi \boldsymbol{f}\right)\right\|_{W^{1, p}\left(B_{R+1}\right)} \leq C_{p, R}\|\boldsymbol{f}\|_{L^{p}\left(\mathbb{R}^{n}\right)} .
\end{aligned}
$$

For $\boldsymbol{f} \in \boldsymbol{L}_{R}^{p}\left(\mathbb{R}^{n}\right)$ and $\lambda \in \Sigma_{\epsilon}(0<\epsilon<\pi)$,

$$
\left\|R_{0}(\lambda) \boldsymbol{f}-R_{0}(0) \boldsymbol{f}\right\|_{W^{2, p}\left(B_{R}\right)} \leq C_{p, R}\left(|\log \lambda|^{\epsilon(n)}|\lambda|^{\frac{n}{2}-1}+|\lambda|^{\frac{n}{2}-1}+|\lambda|\right)\|\boldsymbol{f}\|_{L^{p}\left(\mathbb{R}^{n}\right)},
$$

where $\epsilon(n)=1$ when $n$ is even and $=0$ when $n$ is odd.

2.2. Bogovskiu's lemma and the result in a bounded domain. As was mentioned in $\S 1$, to prove Theorem 1.5, we use Iwashita's cut-off procedure. To apply Iwashita's method, we need some results for stationary problem in bounded domain.

$$
\begin{cases}-\Delta \boldsymbol{u}+\nabla \pi=\boldsymbol{f}, \quad \operatorname{div} \boldsymbol{u}=0, & x \in D, \\ \boldsymbol{n} \cdot \boldsymbol{u}=0, \quad \mathcal{T}(\boldsymbol{u}, \pi)=0, & x \in \partial D .\end{cases}
$$

Here and in what follows, $D \subset \mathbb{R}^{n}$ stands for a bounded domain and $\partial D$ its boundary. For (2.2), Solonnikov and Ščadilov [11] and Shibata and the first author [9] obtained the following theorem.

Theorem $2.3([11,9])$. Let $1<p<\infty$. Assume that $\partial D \in C^{2,1}$ and $D$ is not rotationally symmetric. Then for any $\boldsymbol{f} \in \boldsymbol{L}^{p}(\Omega)$, (2.2) admits a unique solution $(\boldsymbol{u}, \pi) \in \boldsymbol{W}^{2, p}(D) \times$ $\hat{W}^{1, p}(D)$ which satisfies

$$
\|\boldsymbol{u}\|_{W^{2, p}(D)}+\|\pi\|_{W^{1, p}(D)} \leq C\|\boldsymbol{f}\|_{L^{p}(D)} .
$$

REMARK 2.4. The reason why we require that $\Omega$ is not rotationally symmetric is that the proofs of [11] and [9] rely on Korn's first inequality.

In order to keep divergence free condition in the cut-off procedure, we apply the well-known Bogovskiı̌'s lemma. To give a precise statement of Bogovskiı̌'s lemma [1], we introduce the function spaces

$$
\dot{W}^{m, p}(D)=\bar{C}_{0}^{\infty}(D){ }^{\|\cdot\|_{W^{m, p}(D)}}, \quad \dot{W}_{a}^{m, p}(D)=\left\{f \in \dot{W}^{m, p}(D) \mid \int_{D} f d x=0\right\} .
$$

Here $D$ stands for a bounded set in $\mathbb{R}^{n}$ with smooth boundary. 
Lemma 2.5 (Bogovskiur [1]). Let $1<p<\infty$ and let $m \in \mathbb{N}_{0}$. Then there exists a bounded linear operator $\mathbb{B}_{D}: \dot{W}_{a}^{m, p}(Q) \rightarrow \dot{\boldsymbol{W}}^{m+1, p}\left(\mathbb{R}^{n}\right)$ such that

$$
\operatorname{supp} \mathbb{B}_{D}[f] \subset D, \quad \operatorname{div} \mathbb{B}_{D}[f]=f \text { in } \mathbb{R}^{n} .
$$

To use Lemma 2.5, we shall use the following lemma.

Lemma 2.6. Let $1<p<\infty, 0<L<R$ and let $\varphi \in C_{0}^{\infty}\left(\mathbb{R}^{n}\right)$ such that $\varphi(x)=1$ for $|x| \leq L$ and $\varphi(x)=0$ for $|x| \geq R$. Then

(i) If $\boldsymbol{u} \in \boldsymbol{W}^{2, p}\left(\mathbb{R}^{n}\right)$ and $\operatorname{div} \boldsymbol{u}=0$ in $\mathbb{R}^{n}$, then $(\nabla \varphi) \cdot \boldsymbol{u} \in \dot{W}_{a}^{2, p}\left(D_{L, R}\right)$.

(ii) If $\boldsymbol{u} \in \boldsymbol{W}^{2, p}(\Omega)$ and $\operatorname{div} \boldsymbol{u}=0$ in $\Omega$ and $\boldsymbol{n} \cdot \boldsymbol{u}=0$ on $\partial \Omega$, then $(\nabla \varphi) \cdot \boldsymbol{u} \in$ $\dot{W}_{a}^{2, p}\left(D_{L, R}\right)$.

3. Sketch of the proofs. In this section, we sketch the proofs of our main results.

3.1. Analysis of the Stokes resolvent problem in an exterior domain. In order to prove Theorem 1.5, we consider the Stokes resolvent problem in $\Omega$ :

$$
\begin{cases}\lambda \boldsymbol{u}-\Delta \boldsymbol{u}+\nabla \pi=\boldsymbol{f}, \quad \operatorname{div} \boldsymbol{u}=0, & x \in \Omega, \\ \boldsymbol{n} \cdot \boldsymbol{u}=0, \quad \mathcal{T}(\boldsymbol{u}, \pi)=0, & x \in \partial \Omega\end{cases}
$$

Here $\lambda \in \Sigma_{\epsilon}$.

The case when $\lambda$ is large is well studied by Shibata and the first author [9], here our main concern is with $|\lambda|$ small. In particular, to prove Theorem 1.5, it is important to get a resolvent expansion when $\lambda$ is close to the origin, like Theorem 2.1. The main purpose of this subsection is to show the following theorem.

TheOREM 3.1. Let $1<p<\infty$. Then there exists a $\lambda_{0}>0$ such that

$$
(\lambda+A)^{-1} P \boldsymbol{f}=\lambda^{\frac{n}{2}-1}(\log \lambda)^{\epsilon(n)} H_{0}(\lambda)+\lambda^{\frac{n}{2}-1} H_{1}(\lambda)+H_{2}(\lambda)
$$

for any $\lambda \in \dot{U}_{\lambda_{0}}$, where $\epsilon(n)=1$ when $n$ is even and $\epsilon(n)=0$ when $n$ is odd, and $H_{0}(\lambda), H_{1}(\lambda) \in B \mathcal{A}\left(\dot{U}_{\lambda_{0}}, \mathcal{L}_{p, R}(\Omega)\right)$ and $H_{2}(\lambda) \in \mathcal{A}\left(U_{\lambda_{0}}, \mathcal{L}_{p, R}(\Omega)\right)$. Here and hereafter $\mathcal{L}_{p, R}(\Omega) \equiv \mathcal{L}\left(\boldsymbol{L}_{R}^{p}(\Omega), \boldsymbol{W}^{2, p}\left(\Omega_{R}\right)\right)$.

To prove Theorem 3.1, first we shall construct a parametrix to (RP). Fix a positive real number $R>L_{0}$ and let $D$ be an open, bounded, and non-rotationally symmetric set such that $D \supset B_{R+3}$. Then, $\Omega_{D} \equiv \Omega \cap D$ also becomes a non-rotationally symmetric and bounded domain, and $\Omega_{D} \supset \Omega_{R+3} \equiv \Omega \cap B_{R+3}$. Given $\boldsymbol{f} \in \boldsymbol{L}_{R+3}^{p}$, let $\boldsymbol{f}_{0}$ denote the zero extension of $\boldsymbol{f}$ to $\mathbb{R}^{n}: \boldsymbol{f}_{0}=\boldsymbol{f}, x \in \Omega$ and $\boldsymbol{f}_{0}=0, x \notin \Omega$, and let $\boldsymbol{f}_{d}$ denote the restriction of $\boldsymbol{f}$ to $\Omega_{D}$. Let $R_{0}(\lambda)$ and $\Pi$ denote the operators defined in Lemma 2.1.

Since $\Omega_{D}$ is a non-rotationally symmetric bounded domain, in view of Theorem 2.3, let $(\boldsymbol{v}, \theta) \in \boldsymbol{W}^{2, p}\left(\Omega_{D}\right) \times \hat{W}^{1, p}\left(\Omega_{D}\right)$ be a solution to the boundary value problem

$$
\begin{cases}-\Delta \boldsymbol{v}+\nabla \theta=\boldsymbol{f}_{d}, \quad \operatorname{div} \boldsymbol{v}=0, & x \in \Omega_{D}, \\ \boldsymbol{n} \cdot \boldsymbol{v}=0, \quad \mathcal{T}(\boldsymbol{v}, \theta)=0, & x \in \partial \Omega_{D} .\end{cases}
$$

Here $\partial \Omega_{D} \equiv \partial \Omega \cup \partial D$ and $\boldsymbol{n}$ denotes the unit normal vector on $\partial \Omega_{D}$. 
If $\theta$ satisfies (3.1), then $\theta+c$ also satisfies (3.1) for any constant $c \in \mathbb{R}$. Therefore, we may choose a constant $c$ in such a way that

$$
\int_{\Omega_{D}}\left(\theta+c-\Pi \boldsymbol{f}_{0}\right) d x=0 .
$$

Since $\Omega_{D}$ is bounded, $\theta \in \hat{W}^{1, p}\left(\Omega_{D}\right)$ implies that $\int_{\Omega_{D}} \theta d x=0$. Therefore,

$$
c=\frac{1}{\left|\Omega_{D}\right|} \int_{\Omega_{D}} \Pi \boldsymbol{f}_{0} d x,
$$

where $\left|\Omega_{D}\right|$ denotes the Lebesgue measure of $\Omega_{D}$. Let us define operators $A$ and $B$ by relations: $A \boldsymbol{f}=\boldsymbol{v}$ and $B \boldsymbol{f}=\theta+c$. Summing up the above, $A \boldsymbol{f}$ and $B \boldsymbol{f}$ satisfy

$$
\begin{aligned}
& \begin{cases}-\Delta A \boldsymbol{f}+\nabla B \boldsymbol{f}=\boldsymbol{f}_{d}, \quad \operatorname{div} A \boldsymbol{f}=0, & x \in \Omega_{D}, \\
\boldsymbol{n} \cdot A \boldsymbol{f}=0, \quad \mathcal{T}(A \boldsymbol{f}, B \boldsymbol{f})=0, & x \in \partial \Omega_{D},\end{cases} \\
& \int_{\Omega_{D}}\left(B \boldsymbol{f}-\Pi \boldsymbol{f}_{0}\right) d x=0
\end{aligned}
$$

In order to estimate $B \boldsymbol{f}$, we apply the generalized Poincaré inequality:

$$
\|\pi\|_{L^{p}\left(\Omega_{D}\right)} \leq C\left(\|\nabla \pi\|_{L^{p}\left(\Omega_{D}\right)}+\left|\int_{\Omega_{D}} \pi d x\right|\right)
$$

for any $\pi \in W^{1, p}\left(\Omega_{D}\right)$. By Theorem 2.2, Theorem 2.3, (3.2) and (3.3), we obtain $A \in$ $\mathcal{L}\left(\boldsymbol{L}_{R+3}^{p}(\Omega), \boldsymbol{W}^{2, p}\left(\Omega_{D}\right)\right), B \in \mathcal{L}\left(\boldsymbol{L}_{R+3}^{p}(\Omega), W^{1, p}\left(\Omega_{D}\right)\right)$ and

$$
\|A \boldsymbol{f}\|_{W^{2, p}\left(\Omega_{D}\right)}+\|B \boldsymbol{f}\|_{W^{1, p}\left(\Omega_{D}\right)} \leq C\|\boldsymbol{f}\|_{L^{p}(\Omega)} .
$$

Let $\varphi \in C^{\infty}\left(\mathbb{R}^{n}\right), 0 \leq \varphi \leq 1$ and $\varphi=1$ for $|x| \leq R+1$ and $\varphi=0$ for $|x| \geq R+2$. Define operators $\Phi_{\lambda}$ and $\Psi$ by

$$
\begin{aligned}
\Phi_{\lambda} \boldsymbol{f} & =(1-\varphi) R_{0}(\lambda) \boldsymbol{f}_{0}+\varphi A \boldsymbol{f}+\mathbb{B}\left[(\nabla \varphi) \cdot\left(R_{0}(\lambda) \boldsymbol{f}_{0}-A \boldsymbol{f}\right)\right], \\
\Psi \boldsymbol{f} & =(1-\varphi) \Pi \boldsymbol{f}_{0}+\varphi B \boldsymbol{f}
\end{aligned}
$$

for $\boldsymbol{f} \in \boldsymbol{L}_{R+3}^{p}(\Omega)$. Here, in order to apply the Bogovskiı̌'s operator $\mathbb{B}_{D_{R+1, R+2}}$ introduced in Lemma 2.5, we have used the facts that

$$
(\nabla \varphi) \cdot R_{0}(\lambda) \boldsymbol{f}_{0}, \quad(\nabla \varphi) \cdot A \boldsymbol{f} \in \dot{W}^{2, p}\left(D_{R+1, R+2}\right),
$$

which follow from Lemma 2.6. By Lemma 2.5, we obtain

$$
\left\{\begin{array}{l}
\mathbb{B}\left[(\nabla \varphi) \cdot\left(R_{0}(\lambda) \boldsymbol{f}_{0}-A \boldsymbol{f}\right)\right] \in \boldsymbol{W}^{3, p}\left(\mathbb{R}^{3}\right), \\
\operatorname{div} \mathbb{B}\left[(\nabla \varphi) \cdot\left(R_{0}(\lambda) \boldsymbol{f}_{0}-A \boldsymbol{f}\right)\right]=(\nabla \varphi) \cdot\left(R_{0}(\lambda) \boldsymbol{f}_{0}-A \boldsymbol{f}\right), \\
\operatorname{supp} \mathbb{B}\left[(\nabla \varphi) \cdot\left(R_{0}(\lambda) \boldsymbol{f}_{0}-A \boldsymbol{f}\right)\right] \subset D_{R+1, R+2} .
\end{array}\right.
$$

Here and hereafter, we use the abbreviation $\mathbb{B} \equiv \mathbb{B}_{D_{R+1, R+2}}$.

Substituting $\left(\Phi_{\lambda} \boldsymbol{f}, \Psi \boldsymbol{f}\right)$ defined in (3.5) into (RP), we obtain

$$
\begin{cases}(\lambda-\Delta) \Phi_{\lambda} \boldsymbol{f}+\nabla \Psi \boldsymbol{f}=\boldsymbol{f}+S_{\lambda} \boldsymbol{f}, \quad \operatorname{div} \Phi_{\lambda} \boldsymbol{f}=0, & x \in \Omega, \\ \boldsymbol{n} \cdot \Phi_{\lambda} \boldsymbol{f}=0, \quad \mathcal{T}\left(\Phi_{\lambda} \boldsymbol{f}, \Psi \boldsymbol{f}\right)=0, & x \in \partial \Omega .\end{cases}
$$

where

$$
\begin{array}{r}
S_{\lambda} \boldsymbol{f}=2 \nabla \varphi \cdot \nabla\left(R_{0}(\lambda) \boldsymbol{f}_{0}-A \boldsymbol{f}\right)+(\Delta \varphi)\left(R_{0}(\lambda) \boldsymbol{f}_{0}-A \boldsymbol{f}\right)+\lambda A \boldsymbol{f} \\
-(\nabla \varphi)\left(\Pi \boldsymbol{f}_{0}-B \boldsymbol{f}\right)+(\lambda-\Delta) \mathbb{B}\left[(\nabla \varphi) \cdot\left(R_{0}(\lambda) \boldsymbol{f}_{0}-A \boldsymbol{f}\right)\right]
\end{array}
$$


From Lemma 2.1, (3.4) and (3.7), we see that $S_{\lambda} \boldsymbol{f} \in \boldsymbol{W}^{1, p}(\Omega)$ and $\operatorname{supp} S_{\lambda} \boldsymbol{f} \subset \Omega_{R+2}$. Therefore, $S_{\lambda}$ is a compact operator on $\boldsymbol{L}_{R+3}^{p}(\Omega)$. Since

$$
\begin{aligned}
S_{\lambda} \boldsymbol{f}-S_{0} \boldsymbol{f}= & 2 \nabla \varphi \cdot \nabla\left(R_{0}(\lambda) \boldsymbol{f}_{0}-R_{0}(0) \boldsymbol{f}_{0}\right)+(\Delta \varphi)\left(R_{0}(\lambda) \boldsymbol{f}_{0}-R_{0}(0) \boldsymbol{f}_{0}\right)+\lambda A \boldsymbol{f} \\
& +\lambda \mathbb{B}\left[(\nabla \varphi) \cdot\left(R_{0}(\lambda) \boldsymbol{f}_{0}-A \boldsymbol{f}\right)\right]-\Delta \mathbb{B}\left[(\nabla \varphi) \cdot\left(R_{0}(\lambda) \boldsymbol{f}_{0}-R_{0}(0) \boldsymbol{f}_{0}\right)\right],
\end{aligned}
$$

from Lemmas 2.1 and 2.2, (3.4), and Lemma 2.5, we have

$$
\left\|S_{\lambda} \boldsymbol{f}-S_{0} \boldsymbol{f}\right\|_{W^{2, p}\left(\Omega_{R}\right)} \leq C_{p, R}|\lambda|^{\frac{1}{2}}\|\boldsymbol{f}\|_{L^{p}(\Omega)}
$$

provided $\boldsymbol{f} \in \boldsymbol{L}_{R+3}^{p}(\Omega)$ and $\lambda \in \dot{U}_{1 / 2}$.

Our task here is to show that $\left(I+S_{\lambda}\right)$ has the inverse operator and to show that the inverse operator $\left(I+S_{\lambda}\right)^{-1}$ has the expansion of the same type of $R_{0}(\lambda)$ when $\lambda \rightarrow 0$ in $\Sigma_{\epsilon}$. To do so, we shall show that $\left(I+S_{0}\right)^{-1} \in \mathcal{L}\left(\boldsymbol{L}_{R+3}^{p}(\Omega)\right)$.

Since $S_{0}$ is a compact operator on $\boldsymbol{L}_{R+3}^{p}(\Omega)$, by the Fredholm alternative theorem, it is sufficient to show that $I+S_{0}$ is injective. So, let $\boldsymbol{f} \in \boldsymbol{L}_{R+3}^{p}(\Omega)$ be a non-trivial solution of

$$
\left(I+S_{0}\right) \boldsymbol{f}=0 .
$$

From (3.8) with $\lambda=0$ and (3.10), we obtain

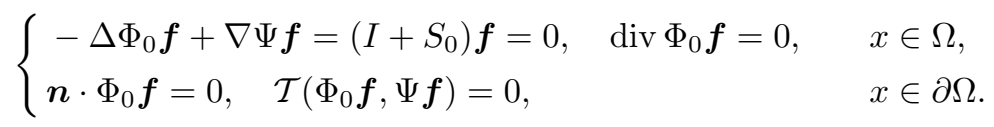

We shall show that $\boldsymbol{f} \equiv 0$ in $\Omega$.

From the definition of $\Phi_{0}$ and $\Psi_{0}$ and Lemma 2.2 , we see that $\left(\Phi_{0} \boldsymbol{f}, \Psi \boldsymbol{f}\right) \in \boldsymbol{W}_{\text {loc }}^{2, p}(\bar{\Omega}) \times$ $W_{\mathrm{loc}}^{1, p}(\bar{\Omega})$ and $\Phi_{0} \boldsymbol{f}=R_{0} \boldsymbol{f}_{0}$ and $\Psi \boldsymbol{f}=\Pi \boldsymbol{f}_{0}$ for $|x| \geq R+2$, and

$$
\nabla^{j} \Phi_{0} \boldsymbol{f}=O\left(|x|^{2-n-j}\right), \quad j=0,1 ; \quad \Psi \boldsymbol{f}=O\left(|x|^{1-n}\right)
$$

In order to conclude that $\boldsymbol{f}=0$, the next lemma concerning uniqueness plays a crucial role.

Lemma 3.2. Let $1<p<\infty$. Let $(\boldsymbol{u}, \pi) \in \boldsymbol{W}_{\text {loc }}^{2, p}(\bar{\Omega}) \times W_{\text {loc }}^{1, p}(\bar{\Omega})$ solve the homogeneous boundary value problem of Stokes equations:

$$
\begin{cases}-\Delta \boldsymbol{u}+\nabla \pi=0, \quad \operatorname{div} \boldsymbol{u}=0, & x \in \Omega, \\ \boldsymbol{n} \cdot \boldsymbol{u}=0, \quad \mathcal{T}(\boldsymbol{u}, \pi)=0, & x \in \partial \Omega,\end{cases}
$$

and satisfy the following radiation conditions:

$$
\nabla^{j} \boldsymbol{u}=O\left(|x|^{2-n-j}\right) \quad j=0,1 ; \quad \pi=O\left(|x|^{1-n}\right) .
$$

Then we have $(\boldsymbol{u}, \pi) \equiv(0,0)$.

Proof. By the boot-strap argument, we may take $(\boldsymbol{u}, \pi) \in \boldsymbol{W}_{\text {loc }}^{2, r}(\bar{\Omega}) \times W_{\text {loc }}^{1, r}(\bar{\Omega})$ for any $r \in(1, \infty)$. In particular, we take $(\boldsymbol{u}, \pi) \in \boldsymbol{W}_{\text {loc }}^{2,2}(\bar{\Omega}) \times W_{\text {loc }}^{1,2}(\bar{\Omega})$. Let $\chi \in C^{\infty}\left(\mathbb{R}^{n}\right)$, $0 \leq \chi \leq 1$ and

$$
\chi= \begin{cases}1, & |x| \leq 1 / 2 \\ 0, & |x| \geq 1\end{cases}
$$


Set $\chi_{R}(x)=\chi(x / R)$. Multiplying the 1st equation of (3.11) by $\chi_{R} \boldsymbol{u}$ and using Green's first identity, we have

$$
\begin{aligned}
0= & \int_{\Omega}(-\Delta \boldsymbol{u}+\nabla \pi) \cdot \chi_{R} \boldsymbol{u} d x \\
= & \int_{\partial \Omega_{R}} \mathbf{T}(\boldsymbol{u}, \pi) \boldsymbol{n} \cdot \chi_{R} \boldsymbol{u} d \sigma+2 \int_{\Omega_{R}} \mathbf{D}(\boldsymbol{u}): \mathbf{D}\left(\chi_{R} \boldsymbol{u}\right) d x-\int_{\Omega_{R}} \pi \operatorname{div}\left(\chi_{R} \boldsymbol{u}\right) d x \\
= & \int_{\partial \Omega_{R}} \chi_{R}(\boldsymbol{n} \cdot \mathbf{T}(\boldsymbol{u}, \pi) \boldsymbol{n}) \boldsymbol{n} \cdot \boldsymbol{u} d \sigma \\
& \quad+2 \int_{\Omega_{R}} \mathbf{D}(\boldsymbol{u}): \mathbf{D}\left(\chi_{R} \boldsymbol{u}\right) d x-\int_{\Omega_{R}} \pi \operatorname{div}\left(\chi_{R} \boldsymbol{u}\right) d x \\
& =2 \int_{\Omega_{R}} \chi_{R} \mathbf{D}(\boldsymbol{u}): \mathbf{D}(\boldsymbol{u}) d x+2 \int_{\Omega_{R}} \mathbf{D}(\boldsymbol{u}):\left(\boldsymbol{u} \otimes \nabla \chi_{R}+{ }^{t}\left(\boldsymbol{u} \otimes \nabla \chi_{R}\right)\right) d x
\end{aligned}
$$

Here $\boldsymbol{u} \otimes \boldsymbol{v} \equiv\left(u_{i} v_{j}\right)_{i, j=1, \ldots, n}$ and we have used the boundary conditions: $\mathbf{T}(\boldsymbol{u}, \pi) \boldsymbol{n}=$ $\boldsymbol{n} \cdot(\boldsymbol{n} \cdot \mathbf{T}(\boldsymbol{u}, \pi) \boldsymbol{n})$ and $\boldsymbol{n} \cdot \boldsymbol{u}=0$ on $\partial \Omega$.

Since $(\boldsymbol{u}, \pi)$ satisfy $(3.12)$, letting $R \rightarrow \infty$ in (3.13), we obtain $\|\mathbf{D}(\boldsymbol{u})\|_{L^{2}(\Omega)}=0$. Hence we have $\mathbf{D}(\boldsymbol{u})=0$, this implies that $\boldsymbol{u}$ is represented as $\boldsymbol{u}(x)=\boldsymbol{A} x+\boldsymbol{b}$, where $\boldsymbol{A}$ is an $n \times n$ anti-symmetric matrix with $\operatorname{tr} \boldsymbol{A}=0$ and $\boldsymbol{b}$ is a constant vector. On the other hand, $\boldsymbol{u}$ satisfies (3.12), $\boldsymbol{A} \equiv 0$ and $\boldsymbol{b} \equiv 0$, hence $\boldsymbol{u} \equiv 0$ in $\Omega$. Substituting $\boldsymbol{u}=0$ into (3.11), we have $\nabla \pi=0$. Therefore $\pi$ is constant. However, $\pi$ should also satisfy (3.12), so $\pi \equiv 0$ in $\Omega$. This completes the proof.

From Lemma 3.2, we have

$$
\left\{\begin{aligned}
\Phi_{0} \boldsymbol{f} & =(1-\varphi) R_{0}(0) \boldsymbol{f}_{0}+\varphi A \boldsymbol{f}+\mathbb{B}\left[(\nabla \varphi) \cdot\left(R_{0}(0) \boldsymbol{f}_{0}-A \boldsymbol{f}\right)\right]=0, & & \text { in } \Omega, \\
\Psi \boldsymbol{f} & =(1-\varphi) \Pi \boldsymbol{f}_{0}+B \boldsymbol{f}=0, & & \text { in } \Omega .
\end{aligned}\right.
$$

Since $\varphi=1$ for $|x| \leq R+1$ and $\varphi=0$ for $|x| \geq R+2$ and $\operatorname{supp} \mathbb{B}\left[(\nabla \varphi) \cdot\left(R_{0}(0) \boldsymbol{f}_{0}-A \boldsymbol{f}\right)\right] \subset$ $D_{R+1, R+2}$, we have

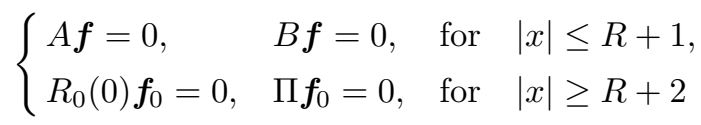

Set

$$
\boldsymbol{w}=\left\{\begin{array}{ll}
A \boldsymbol{f}, & x \in \Omega_{D}, \\
0, & x \notin \Omega,
\end{array} \quad \psi= \begin{cases}B \boldsymbol{f}, & x \in \Omega_{D}, \\
0, & x \notin \Omega\end{cases}\right.
$$

By (3.6) and (3.15), $(\boldsymbol{w}, \psi) \in \boldsymbol{W}^{2, p}(D) \times W^{1, p}(D)$ and satisfy

$$
\begin{cases}-\Delta \boldsymbol{w}+\nabla \psi=\boldsymbol{f}_{0}, \quad \operatorname{div} \boldsymbol{w}=0, & x \in D \\ \boldsymbol{n} \cdot \boldsymbol{w}=0, \quad \mathcal{T}(\boldsymbol{w}, \psi)=0, & x \in \partial D .\end{cases}
$$

On the other hand, by $(3.15),\left(R_{0}(0) \boldsymbol{f}_{0}, \Pi \boldsymbol{f}_{0}\right)$ also satisfy

$$
\begin{cases}-\Delta R_{0}(0) \boldsymbol{f}_{0}+\nabla \Pi \boldsymbol{f}_{0}=0, \quad \operatorname{div} R_{0}(0) \boldsymbol{f}_{0}=0, & x \in D, \\ \boldsymbol{n} \cdot R_{0}(0) \boldsymbol{f}_{0}=0, \quad \mathcal{T}\left(R_{0}(0) \boldsymbol{f}_{0}, \Pi \boldsymbol{f}_{0}\right)=0, & x \in \partial D\end{cases}
$$

Since $D$ is a non-rotationally symmetric bounded domain, by virtue of Theorem 2.3 , we have $\boldsymbol{w}=R_{0}(0) \boldsymbol{f}_{0}, \psi=\Pi \boldsymbol{f}_{0}+c$ with some constant $c \in \mathbb{R}$. Therefore $A \boldsymbol{f}=R_{0}(0) \boldsymbol{f}_{0}$ 
and $B \boldsymbol{f}-\Pi \boldsymbol{f}_{0}=c$ in $\Omega_{D}$. From (3.2), we have $c=0$, which yields $B \boldsymbol{f}=\Pi \boldsymbol{f}_{0}$ in $\Omega_{D}$. Combining these facts with (3.14), we see that

$$
\begin{gathered}
\mathbb{B}\left[(\nabla \varphi) \cdot\left(R_{0}(0) \boldsymbol{f}_{0}-A \boldsymbol{f}\right)\right]=0, \quad x \in \mathbb{R}^{n}, \\
0=(1-\varphi) R_{0}(0) \boldsymbol{f}_{0}+\varphi A \boldsymbol{f}=R_{0}(0) \boldsymbol{f}_{0}+\varphi\left(A \boldsymbol{f}-R_{0}(0) \boldsymbol{f}_{0}\right)=R_{0}(0) \boldsymbol{f}_{0}, \quad x \in \Omega \\
0=(1-\varphi) \Pi \boldsymbol{f}_{0}+\varphi B \boldsymbol{f}=\Pi \boldsymbol{f}_{0}+\varphi\left(B \boldsymbol{f}-\Pi \boldsymbol{f}_{0}\right)=\Pi \boldsymbol{f}_{0}, \quad x \in \Omega .
\end{gathered}
$$

Therefore, we obtain

$$
0=-\Delta R_{0}(0) \boldsymbol{f}_{0}+\nabla \Pi \boldsymbol{f}_{0}=\boldsymbol{f}_{0},
$$

which implies that $\boldsymbol{f}=0$ in $\Omega$. This completes the proof of $\boldsymbol{f} \equiv 0$. Therefore from the Fredholm alternative theorem, we can conclude that $\left(I+S_{0}\right)^{-1} \in \mathcal{L}\left(\boldsymbol{L}_{R+3}^{p}(\Omega)\right)$ exists.

Let $M=\|\|\left(I+S_{0}\right)^{-1} \mid \|$. Here and hereafter \|\|$\cdot \|$ denotes the operator norm of $\mathcal{L}\left(\boldsymbol{L}_{R+3}^{p}(\Omega)\right)$. Since

$$
I+S_{\lambda}=\left(I+S_{0}\right)\left(I+\left(I+S_{0}\right)^{-1}\left(S_{\lambda}-S_{0}\right)\right)
$$

if we choose $\lambda_{0}>0$ so small that $\left\|\left|S_{\lambda}-S_{0} \|\right|<1 / 2 M\right.$ for $\lambda \in \dot{U}_{1 / 2}$, we have

$$
\left(I+S_{\lambda}\right)^{-1}=\left(\sum_{k=0}^{\infty}\left[\left(I+S_{0}\right)^{-1}\left(S_{\lambda}-S_{0}\right)\right]^{k}\right)\left(I+S_{0}\right)^{-1}
$$

for any $\lambda \in \dot{U}_{\lambda_{0}}$, where we have used (3.9).

Set $R(\lambda) \boldsymbol{f}=\Phi_{\lambda}\left(I+S_{\lambda}\right)^{-1} \boldsymbol{f}$ and $\Pi(\lambda) \boldsymbol{f}=\Psi\left(I+S_{\lambda}^{-1}\right) \boldsymbol{f}$ for $\boldsymbol{f} \in \boldsymbol{L}_{R+3}^{p}(\Omega)$ and $\lambda \in \dot{U}_{\lambda_{0}}$. Then, by (3.8) we have

$$
\begin{cases}(\lambda-\Delta) R(\lambda) \boldsymbol{f}+\nabla \Pi(\lambda) \boldsymbol{f}=\boldsymbol{f}, \quad \operatorname{div} R(\lambda) \boldsymbol{f}=0, & x \in \Omega, \\ \boldsymbol{n} \cdot R(\lambda)=0, \quad \mathcal{T}(R(\lambda) \boldsymbol{f}, \Pi(\lambda) \boldsymbol{f})=0, & x \in \partial \Omega,\end{cases}
$$

which combined with the Helmholtz decomposition gives $\left(\lambda+A_{p}\right) R(\lambda) \boldsymbol{f}=P_{p} \boldsymbol{f}$ in $\Omega$. This implies that

$$
\left(\lambda+A_{p}\right)^{-1} P_{p} \boldsymbol{f}=R(\lambda) \boldsymbol{f}
$$

provided that $\lambda \in \dot{U}_{\lambda_{0}}$ and $\boldsymbol{f} \in \boldsymbol{L}_{R+3}^{p}(\Omega)$. The asymptotic expansion of the resolvent follows from the definition of $R(\lambda): R(\lambda)=\Phi_{\lambda}\left(I+S_{\lambda}\right)^{-1}$ and Lemma 2.1. This completes the proof of Theorem 3.1.

3.2. Proof of Theorem 1.5. Finally we shall prove the local energy decay. Let $t \geq 1$ and take $\epsilon_{0}$ and $\delta_{0}$ such that $0<\epsilon_{0}<\pi / 2$ and $0<\delta_{0}<\lambda_{0}$, where $\lambda_{0}$ is the same constant as in Theorem 3.1. Let $\gamma$ be a contour so that $\gamma=\gamma_{1} \cup \gamma_{2}$, where

$$
\gamma_{1}=\left\{z \in \mathbb{C}|| z\left|\leq \delta_{0},\right| \arg z \mid=\epsilon_{0}\right\}, \gamma_{2}=\left\{z \in \mathbb{C}|| z\left|\geq \delta_{0},\right| \arg z \mid=\epsilon_{0}\right\} .
$$

The Stokes semigroup $e^{-t A}$ can be represented as

$$
e^{-t A_{p}} P_{p} \boldsymbol{f}=\frac{1}{2 \pi i} \int_{\gamma_{1}} e^{\lambda t} R(\lambda) \boldsymbol{f} d \lambda+\frac{1}{2 \pi i} \int_{\gamma_{2}} e^{\lambda t}\left(\lambda+A_{p}\right)^{-1} P_{p} \boldsymbol{f} d \lambda \equiv J_{1}(t)+J_{2}(t)
$$

for $\boldsymbol{f} \in \boldsymbol{L}_{R}^{p}(\Omega)$. We shall estimate the $W^{2, p}$-norm of $J_{1}(t)$ and $J_{2}(t)$ over $\Omega_{R}$ separately.

First we shall estimate $J_{2}(t)$. We use the resolvent estimate obtained by Shibata and Shimada [9]:

$$
|\lambda|\|\boldsymbol{u}\|_{L^{p}(\Omega)}+\|\boldsymbol{u}\|_{W^{2, p}(\Omega)} \leq C\|\boldsymbol{f}\|_{L^{p}(\Omega)}
$$


for any $\boldsymbol{f} \in L_{\sigma}^{p}(\Omega)$ and $\lambda \in \Sigma_{\epsilon}$ with $|\lambda| \geq \delta_{0}$. By (3.16) and $L^{p}$-boundedness of the Helmholtz projection $P_{p}$, we have

$$
\left\|J_{2}(t)\right\|_{W^{2, p}\left(\Omega_{R}\right)} \leq C \int_{\delta_{0}}^{\infty} e^{t \cos \left(\pi-\epsilon_{0}\right) r} d r\left\|P_{p} \boldsymbol{f}\right\|_{L^{p}(\Omega)} \leq C \frac{e^{-t\left(\cos \epsilon_{0}\right) \delta_{0}}}{t \cos \epsilon_{0}}\|\boldsymbol{f}\|_{L^{p}(\Omega)} .
$$

From Theorem 3.1, we can split $J_{1}(t)$ into the following three terms:

$$
\begin{aligned}
J_{1}(t)= & \frac{1}{2 \pi i} \int_{\gamma_{1}} e^{\lambda t} \lambda^{\frac{n}{2}-1}(\log \lambda)^{\epsilon(n)} H_{0}(\lambda) \boldsymbol{f} d \lambda \\
& \quad+\frac{1}{2 \pi i} \int_{\gamma_{1}} e^{\lambda t} \lambda^{\frac{n}{2}-1} H_{1}(\lambda) \boldsymbol{f} d \lambda+\frac{1}{2 \pi i} \int_{\gamma_{1}} e^{\lambda t} H_{2}(\lambda) \boldsymbol{f} d \lambda \\
\equiv & J_{1,0}(t)+J_{1,1}(t)+J_{1,2}(t) .
\end{aligned}
$$

Since $H_{1}(\lambda) \in B \mathcal{A}\left(U_{\lambda_{0}}, \mathcal{L}_{p, R}(\Omega)\right)$, we have

$$
\begin{aligned}
\left\|J_{1,1}(t)\right\|_{W^{2, p}\left(\Omega_{R}\right)} & \leq C \int_{0}^{\delta_{0}} e^{\operatorname{Re} \lambda t}|\lambda|^{\frac{n}{2}-1}|d \lambda|\|\boldsymbol{f}\|_{L^{p}(\Omega)} \\
& <C \int_{0}^{\infty} e^{-\left(r \cos \epsilon_{0}\right) t} r^{\frac{n}{2}-1} d r \\
& \leq C t^{-\frac{n}{2}} \int_{0}^{\infty} e^{-s \cos \epsilon_{0}} s^{\frac{n}{2}-1} d s \leq C t^{-\frac{n}{2}} .
\end{aligned}
$$

Here we have used the change of variables: $s=r t$ and the fact that $\epsilon_{0} \in(0, \pi / 2)$.

Next we shall estimate $J_{1,2}(t)$. Since $H_{2}(\lambda) \in \mathcal{A}\left(U_{\lambda_{0}}, \mathcal{L}_{p, R}(\Omega)\right)$, changing $\gamma_{1}$ to the path: $z=-\delta_{0} \cos \epsilon_{0}+i s\left(|s| \leq \delta_{0} \sin \epsilon_{0}\right)$, by Cauchy's integral theorem, we have

$$
\begin{aligned}
\left\|J_{1,2}(t)\right\|_{W^{2, p}\left(\Omega_{R}\right)} & \leq C \int_{-\delta_{0} \sin \epsilon_{0}}^{\delta_{0} \sin \epsilon_{0}} e^{-\left(\delta_{0} \cos \epsilon_{0}\right) t} d s\|\boldsymbol{f}\|_{L^{p}(\Omega)} \\
& \leq 2 C \delta_{0} \sin \epsilon_{0} e^{-\left(\delta_{0} \cos \epsilon_{0}\right) t}\|\boldsymbol{f}\|_{L^{p}(\Omega)} .
\end{aligned}
$$

Finally we shall estimate $J_{0,1}(t)$ when $n \geq 4$ is even. We apply the well known formula for the gamma function $\Gamma(\sigma)$.

LEMma 3.3. Let $j$ be a non-negative integer and $\sigma>0$. For any $t>0$,

$$
\frac{1}{2 \pi i} \int_{\gamma} e^{\lambda t} \lambda^{j} \log \lambda d \lambda=\left.\frac{d}{d \sigma}\left[\frac{\sin \sigma \pi}{\pi} \Gamma(\sigma) e^{i \pi \sigma}\right]\right|_{\sigma=j+1} t^{-(j+1)} .
$$

Since $n$ is even, applying Lemma 3.3 with $j=\frac{n}{2}-1 \in \mathbb{N}$, we obtain

$$
\left\|J_{1,0}(t)\right\|_{W^{2, p}\left(\Omega_{R}\right)} \leq C t^{-\frac{n}{2}}\|\boldsymbol{f}\|_{L^{p}(\Omega)} .
$$

Combining (3.17)-(3.20), the proof of Theorem 1.5 is completed.

3.3. $L^{p}-L^{q}$ estimate for the Stokes semigroup. In this subsection, we shall give a sketch of the proof of Theorem 1.2. The basic idea is similar to that of Iwashita [4] (see also Kobayashi and Shibata [6], Hishida [3] and Yamaguchi [13] and references therein).

Given $\boldsymbol{f} \in L_{\sigma}^{p}(\Omega)$, we set $\boldsymbol{u}(t)=e^{-t A_{p}} \boldsymbol{f}$. Then $\boldsymbol{u}(t)$ solves the nonstationary Stokes equations under the free slip boundary condition with suitable pressure term $\pi=\pi(t)$.

In order to show Theorem 1.2, as a first step, we shall derive a sharp local energy decay estimate for $\boldsymbol{f} \in L_{\sigma}^{p}(\Omega)$. 
Lemma 3.4. Let $1<p<\infty$ and $R>L_{0}+1$. Then there exists a positive constant such that

$$
\left\|\partial_{t} e^{-t A_{p}} \boldsymbol{f}\right\|_{W^{1, p}\left(\Omega_{R}\right)}+\left\|e^{-t A_{p}} \boldsymbol{f}\right\|_{W^{2, p}\left(\Omega_{R}\right)} \leq C t^{-\frac{n}{2 p}}\|\boldsymbol{f}\|_{L^{p}(\Omega)}
$$

for any $t \geq 2$ and $\boldsymbol{f} \in L_{\sigma}^{p}(\Omega)$.

The proof of Lemma 3.4 is done by a cut-off procedure based on $L^{p}-L^{q}$ estimates for the heat semigroup $e^{t \Delta}$ in $\mathbb{R}^{n}$ and Theorem 1.2 (local energy decay). We omit the details.

Our next aim is to obtain the following lemma.

Lemma 3.5. Let $1<p<\infty$ and $\boldsymbol{f} \in L_{\sigma}^{p}(\Omega)$. Then

$$
\left\|e^{-t A_{p}} \boldsymbol{f}\right\|_{L^{q}(\Omega)} \leq C_{p, q} t^{-\frac{n}{2}\left(\frac{1}{p}-\frac{1}{q}\right)}\|\boldsymbol{f}\|_{L^{p}(\Omega)} \quad \text { for } t \geq 2
$$

provided that $p \leq q \leq \infty$ and $n(1 / p-1 / q)<2$ and

$$
\left\|\nabla e^{-t A_{p}} \boldsymbol{f}\right\|_{L^{p}(\Omega)} \leq C_{p} t^{-\frac{1}{2}}\|\boldsymbol{f}\|_{L^{p}(\Omega)} \quad \text { for } t \geq 2
$$

provided that $1<p \leq n$.

In view of Lemma 3.4, it suffices to consider the case $\Omega \backslash B_{R}$ and $t \geq 2$. The proof of Lemma 3.5 is carried out by the standard cut-off method and elementary calculations.

From the complex interpolation and Sobolev embedding theorem, we obtain

Lemma 3.6. Let $1<p<\infty, 0<t \leq 2$ and $\boldsymbol{f} \in L_{\sigma}^{p}(\Omega)$. Then

$$
\left\|\nabla^{j} e^{-t A_{p}} \boldsymbol{f}\right\|_{L^{q}(\Omega)} \leq C_{p, q} t^{-\frac{j}{2}-\frac{n}{2}\left(\frac{1}{p}-\frac{1}{q}\right)}\|\boldsymbol{f}\|_{L^{p}(\Omega)}
$$

where (i) $1 \leq p \leq q \leq \infty$ when $j=0$; (ii) $1<p \leq q<\infty$ when $j=1$.

Finally, combining Lemmas 3.5 and 3.6, we obtain Theorem 1.2.

Acknowledgements. The authors would like to express their sincere gratitude to Prof. Yoshihiro Shibata for much valuable advice.

\section{References}

[1] M. E. Bogovskiı̌, Solution of the first boundary value problem for an equation of continuity of an incompressible medium, Dokl. Akad. Nauk SSSR 248 (1979), 1037-1040.

[2] Y. Giga, The nonstationary Navier-Stokes system with some first order boundary condition, Proc. Japan Acad. Ser. A Math. Sci. 58 (1982), 101-104.

[3] T. Hishida, The nonstationary Stokes and Navier-Stokes flows through an aperture, in: Contributions to Current Challenges in Mathematical Fluid Mechanics, Adv. Math. Fluid Mech., Birkhäuser, Basel, 2004, 79-123.

[4] H. Iwashita, $L_{q}-L_{r}$ estimates for solutions of the nonstationary Stokes equations in an exterior domain and the Navier-Stokes initial value problems in $L_{q}$ spaces, Math. Ann. 285 (1989), 265-288.

[5] T. Kato, Strong $L^{p}$-solutions of the Navier-Stokes equation in $\mathbb{R}^{m}$, with applications to weak solutions, Math. Z. 187 (1984), 471-480.

[6] T. Kobayashi and Y. Shibata, On the Oseen equation in the three-dimensional exterior domains, Math. Ann. 310 (1998), 1-45. 
[7] T. Miyakawa, On nonstationary solutions of the Navier-Stokes equations in an exterior domain, Hiroshima Math. J. 12 (1982), 115-140.

[8] J. Saal, Stokes and Navier-Stokes equations with Robin boundary conditions in a halfspace, J. Math. Fluid Mech. 8 (2006), 211-241.

[9] Y. Shibata and R. Shimada, On a generalized resolvent estimate for the Stokes system with Robin boundary condition, J. Math. Soc. Japan 59 (2007), 469-519.

[10] R. Shimada and N. Yamaguchi, Global solution to the Navier-Stokes equations with Navier's slip boundary in exterior domains, preprint.

[11] V. A. Solonnikov and V. E. Ščadilov, A certain boundary value problem for the stationary system of Navier-Stokes equations, Trudy Mat. Inst. Steklova 125 (1973), 196-210.

[12] O. Steiger, Navier-Stokes equations with first order boundary conditions, J. Math. Fluid Mech. 8 (2006), 456-481.

[13] N. Yamaguchi, On an existence theorem of global strong solution to the magnetohydrodynamic system in three dimensional exterior domain, Differential Integral Equations 19 (2006), 919-944. 